\title{
CARACTERIZACIÓN DE SISTEMAS AGROFORESTALES TRADICIONALES EN EL PACIFICO SUR DE COLOMBIA, DEPARTAMENTO DE NARIÑO
}

\author{
Characterization of traditional agroforestal systems in the South Pacific \\ of Colombia, departrament of Nariño
}

\section{Artículo de Investigación}

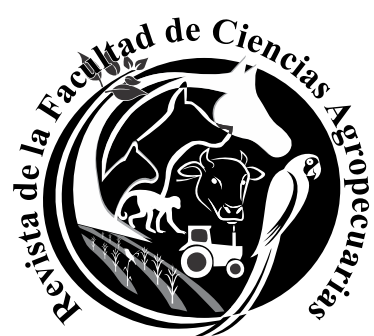

FAGROPEC

Recibido 21 de agosto de 2019. Aceptado 29 de noviembre de 2019.

1. I.A, Profesor Asociado. Facultad Ciencias Agrícolas, Universidad de Nariño.

(iD) https://orcid.org/0000-0002-2441-2400

${ }^{2}$ I.A, Profesor Asociado. Facultad Ciencias Agrícolas, Universidad de Nariño.

(iD) https://orcid.org/0000-0002-4290-1830

${ }^{3}$ I.A. Profesor titular. Facultad Ciencias Agrarias, Universidad de Montería, Córdoba

iD https://orcid.org/0000-0002-3671-2865

\section{Como citar:}

NAVIA ESTRADA, J. F., et al. Caracterización de sistemas agroforestales tradicionales en el Pacifico sur de Colombia, departamento de Nariño. En: Revista Facultad Ciencias Agropecuarias - FAGROPEC. Universidad de la Amazonia, Florencia - Caquetá. Volumen 11 julio-diciembre, 2019. Pp. 90-101 ISSNRevista en Línea: 2539-178X

*Autor para correspondencia: jornavia@gmail.com
Jorge Fernando Navia Estrada ${ }^{1 *}$ Ph.D; Orlando Benavides Benavides ${ }^{2}$ Ph.D y Fernando Vicente Barraza Älvarez ${ }^{3}$ Ph.D

La presente investigación se realizó en el municipio de Tumaco, departamento de Nariño, en el Consejo Comunitario Río Mejicano localizado a $1^{\circ} 40^{\prime} 50^{\prime \prime}$ Latitud Norte y 78³1'11" Longitud Oeste de Greenwich, cuyo objetivo fue caracterizar los sistemas agroforestales tradicionales y proponer opciones agroforestales a partir de la realidad local. Para lo cual, se aplicó una encuesta a los productores que permitieron la identificación de los sistemas productivos tradicionales; El arreglo agroforestal más generalizado en el río Mejicano es el Silvoagrícola, conformado por Cacao (Theobroma cacao) y Plátano (Musa sapientum), frutales y maderable; La especie forestal mas importante en el sistema es el Cedro (Cedrela odorata)y los frutales, que se encontraron en mayor frecuencia fueron los cítricos (Naranja y Limón), que le brinda al sistema características especiales de aroma y sabor. De acuerdo a esto se propone implementar sistemas agroforestales silvoagrícolas basados en las especies de Cacao, Plátano y Maderables, a partir del mejoramiento técnico de los sistemas tradicionales actuales.

Palabras claves:

Asociación; Sistema tipificado; Pacífico.

\section{ABSTRACT}

The present investigation was carried out in the municipality of Tumaco, department of Nariño, in the Mejicano river Community Council located at $1^{\circ} 40^{\prime} 50$ "North Latitude and $78^{\circ} 31^{\prime} 11^{\prime}$ " West Longitude of Greenwich. The objective of the work was to characterize traditional agroforestry systems and propose agroforestry options based on local reality afterwards. For that reason, a survey was applied to the producers that allowed the identification of traditional production systems; The most widespread agroforestry arrangement on the Mejicano river is the Silvoagrícola, that consist of Cocoa (Theobroma cacao) and Banana (Musa sapientum), fruit trees and timber; The most important forest species in the system is the Cedar (Cedrela odorata) and the fruit trees. The most frequently found are citrus fruits (Orange and Lemon), which give the system special aroma and flavor characteristics. According to the results of the investigation, it is proposed to implement agroforestry silvoagricultural systems based on the species of 
Cocoa, Banana and Timber. That can be reached by technical improvement of current traditional systems

Key words:

Association; Typified system; Pacific.

\section{INTRODUCCIÓN}

El municipio de Tumaco, se caracteriza por ser uno de los más extensos del país con un territorio de 360.172 Has, de las cuales, el 48,21\% (173.632 hectáreas) están tituladas colectivamente a favor de las comunidades negras afrodescendientes, reconocidas mediante la Ley 70 de 1993 (Plan de desarrollo Tumaco, 2011). Para acceder a la titulación colectiva, las comunidades negras se organizan en Consejos Comunitarios, que para el caso del municipio de Tumaco, se han constituido un total de 15 alrededor de las cuencas hidrográficas y Uno de estos Consejos Comunitarios es el del Río Mejicano conformado con cuatro (4) veredas, y una extensión total de 13.462 hectáreas y una población estimada de 2.575 habitantes.

En los últimos años los Consejos Comunitarios, con cacao procedente de los sistemas productivos tradicionales, se han ganado reiterativamente los premios a la excelencia en la calidad del cacao en diferentes partes del mundo (Gobernación de Nariño, 2015). Desafortunadamente, los sistemas productivos tradicionales presentan muy baja rentabilidad y poca diversidad, lo que genera bajos ingresos y afectación a la seguridad alimentaria en los Consejos comunitarios.

Las comunidades negras afrodescendientes, desarrollan prácticas tradicionales de producción caracterizadas por el establecimiento de fincas diversificadas que constituyen en la práctica sistemas agroforestales con especies agrícolas (principal cacao), frutales y forestales, que es necesario caracterizar dado que en Colombia y en el sur del país, los trabajos de caracterización e identificación de sistemas tradicionales agroforestales, no han tenido la suficiente investigación, debido a la complejidad de los mismos y al desconocimiento de técnicas de manejo de información. Es así como se desconoce el estado de los agroecosistemas productivos de muchas regiones en las cuales se puede encontrar un gran potencial de recursos, los cuales podrían ser aprovechados de manera más eficiente y sostenible.

CORPOICA (1996), en la caracterización de los sistemas productivos del Pacifico afirma que ésta se puede entender como la determinación del efecto integral de los factores físicos, bióticos, económicos, socioculturales y ambientales, que permiten conocer, entender y formular hipótesis acerca de la estructura, función, manejo y razón de ser de los sistemas de producción en áreas especificas, y aporta elementos de análisis para quienes toman decisiones entorno al desarrollo regional(Navia y Hernández, 1999).

El sistema de producción agrícola, es un ecosistema que cambia, maneja y administra el hombre con el fin de producir bienes que le son útiles para sí mismo (Parlamento Científico de jóvenes, 2016).

Sánchez y Leal (1994), destacan que la economía rural de las comunidades que habitan en el pacifico 
colombiano, han sido muy poco estudiadas y comprendida, muchos investigadores, tanto técnicos como funcionarios de instituciones, han producido documentos e informes sobre los sistemas agrícolas y forestales tradicionales; algunos de ellos no exentos de prejuicios, en la que se desvalorizan las prácticas económicas de las comunidades negras e indígenas, calificadas como atrasadas o primitivas. Reafirmando la tendencia dominante al considerar viables únicamente las actividades extractivas.

A inicios de la década de los años 70, comienzan a cobrar fuerza los estudios sobre el Pacifico colombiano tratando de comprender su lógica interna desde los puntos de vista económico y sociocultural, así lo afirman Corsetti, Motta y Tasara (1990) en diferentes estudios realizados, que tratan sobre los cambios tecnológicos, las organizaciones sociales y las actividades productivas predominantes de la costa pacífica colombiana.

El presente estudio, representa para estas comunidades, una estrategia para la recuperación de las prácticas tradicionales de producción, a partir de su reconocimiento y apropiación por parte de los pobladores del territorio, dado que se han ido perdiendo como consecuencia de los procesos de deterioro progresivo de los recursos naturales y descomposición social de la población, debido a la proliferación de los cultivos de uso ilícito, la presencia de grupos armados ilegales dedicados al narcotráfico, la sobre-explotación de los recursos naturales y las fumigaciones aéreas indiscriminadas con glifosato que afectan los cultivos lícitos tradicionales. Por lo cual el objetivo principal fue caracterizar los sistemas agroforestales presentes en el Río Mejicano.

\section{METODOLOGÍA}

El estudio se realizo en: El territorio del Consejo Comunitario Río Mejicano que se encuentra localizado en las tierras bajas del pacifico sur colombiano en el departamento de Nariño en la microcuenca del Río Mejicano, al noroccidente del municipio de Tumaco, con las coordenadas geográficas 140'50" Latitud Norte y 78³1'11" Longitud Oeste de Greenwich. Para la caracterización de los sistemas de producción agropecuarios tradicionales en la zona del Río Mejicano, se realizó una revisión de fuentes secundarias de información sobre la zona de estudio; para este efecto, se recopiló información en aspectos relacionados con la investigación en entidades gubernamentales y no gubernamentales como CORPONARIÑO, ICA, RECOMPAS y Proyecto ADAM-MONTEBRAVO.

De acuerdo con el Plan de Manejo Ambiental, hecho por la Universidad Nacional, sede Tumaco 2015 (Plan de desarrollo Tumaco, 2018), el territorio se ubica en un rango de precipitación que va desde los 2500 - $3500 \mathrm{~mm}$ anuales. Según los registros de precipitación del municipio de Tumaco, los periodos de menor lluvia se presentan en los meses de agosto, septiembre y noviembre, siendo los más lluviosos, abril mayo y junio. Posee una temperatura máxima de $29^{\circ} \mathrm{C}$, durante el día épocas soleadas, mínima $23^{\circ} \mathrm{C}$ durante la noche en periodos lluviosos y temperatura promedio $26^{\circ} \mathrm{C}$. El aire es húmedo y sus porcentajes más altos se presentan hacia el final de la tarde, durante la noche y las primeras horas de la mañana y los más bajos hacia el mediodía. Su valor medio es del 87\% (PMIA 2003). Por su parte, el brillo solar es relativamente bajo, no supera las 3,5 horas luz /día. Según Holdridge (1982), el territorio pertenece a la zona de vida bosque húmedo tropical (bh-T) y muy húmedo tropical (bmh-T). 
Estimación de la muestra: El número de encuestas a aplicar o muestra, se tomó según la población total de familias presentes en la zona de estudio; se aplicó la fórmula para el muestreo aleatorio estratificado y se determino el número de encuestas a realizar, tal como se describe a continuación:

$$
n \geq \frac{N\left[\sum_{i=1}^{\mathrm{L}}\left(U_{i} S_{i}^{2}\right)\right]}{N^{2}\left[\frac{d}{Z_{1-a / 2}}\right]^{2}+\left[\sum_{i=1}^{\mathrm{L}}\left(U_{i} S_{i}^{2}\right)\right]} \quad \text { Castillo, (2002) }
$$

Donde

$n=$ Tamaño de la muestra común

$N=$ Total de unidades de muestreo en que está dividida la población

$L=$ Número de estratos en que se divide la población

$U_{i}=$ Tamaño de la muestra para el $i$-ésimo estrato

$s^{2}=$ Varianza de la muestra aleatoria simple tomada en el i-ésimo estrato

$s_{i}=$ Desviación estándar de la muestra aleatoria simple tomada en el $i$-ésimo estrato

$w_{i}=$ Importancia del $i$-ésimo estrato

$Z_{1-\alpha / 2}=$ Cuantil de la distribución normal con una distribución menor o igual a $1-\alpha / 2$

$d=$ Máximo error de alejamiento con respecto al verdadero valor del parámetro que el investigador está dispuesto a aceptar.

Análisis estadístico: La información recopilada se sistematizó en Excel por bloques de preguntas; esta información se procesó mediante el análisis de componentes principales (ACP), a través del software SAS V8O, para variables cuantitativas. y El análisis de correspondencias múltiples ACM a través del software SPAD V3.5 para variables cualitativas.

Las variables que se tuvieron en cuenta para ACP fueron: producción de cacao (v1), cantidad de has dedicadas a sistemas agroforestales (v2), cantidad de has en agricultura (v4), cantidad de has en ganadería (v5), costos de producción (v6), número de familias (v8) e ingreso total (v10). Para ACM se tuvieron en cuenta las siguientes variables: fertilidad(v1), disponibilidad de aguas (v2), tipo de sistemas que tiene la finca (v3) problemas fitosanitarios (v4), labores fundamentales (v5), tenencia de tierra (v11), nivel de escolaridad (v12) y especie que mas posee en su finca (v13).

\section{RESULTADOS Y DISCUSIÓN}

\section{Análisis de componentes principales (ACP)}

El análisis de componentes principales permitió establecer, con base en el porcentaje acumulado de los valores propios, un total de dos componentes (tabla, 1), los cuales permiten explicar el 52,19\% de

Tabla 1. Valores propios (\% Varianza) por componente de los sistemas productivos del Consejo Comunitario Río Mexicano, Tumaco.

\begin{tabular}{lccc}
\hline Componentes & Valores propios & \% varianza & \% varianza acumulada \\
\hline Comp. 1 & 1,80 & 33,66 & 33,66 \\
Comp. 2 & 1,40 & 18,52 & 52,19 \\
Comp. 3 & 1,10 & 12,80 & 64,98 \\
Comp. 4 & 0,96 & 9,80 & 74,78 \\
Comp. 5 & 0,70 & 7,60 & 82,38 \\
\hline
\end{tabular}


la variabilidad total de las fincas evaluadas en el Rio Mejicano.

El primer factor permite explicar el 33,66\% de la variabilidad y está conformado principalmente por variables como (tabla 2), producción de Cacao (V1), cantidad de áreas en sistemas agroforestales (V3), cantidad de área en agricultura (V4) y costos de producción, estas variables presentaron una correlación variable factor del orden de $(0.74,0.90,0.80$ y 0.59$)$.

El segundo factor mostró un $18.52 \%$, de la explicación de la variabilidad total de la fincas evaluadas, estuvo conformado principalmente por la variables (tabla 2), número de integrantes de las familias $(\mathrm{V} 8=0.71)$ y el ingreso total $(\mathrm{V} 10=-0.67)$.

También, se presentó alta correlación, al realizar el análisis de las variables cuantitativas seleccionadas por su alta variabilidad, donde se permitió establecer inicialmente altas correlaciones entre la cantidad de hectáreas destinadas a la producción de SAF (V3) y la producción de Cacao (Teobroma cacao L.) (V1) $(0,64)$; y la cantidad de hectáreas en agricultura (V4) con la cantidad de hectáreas en SAF (V3) con una correlación de 0,67.

\section{Análisis de clasificación}

El análisis clasificatorio en n índice de Ward 0.5, permitió agrupar las fincas en cuatro grupos, caracterizado por su afinidad intragrupal y sus diferencias intergrupales figura 1.

El primer grupo : Está conformado por 13 fincas, los cuales representan el 21,66\% de las fincas evaluadas. Este grupo se caracterizó por fincas que presentaron un promedio menor de 64,38, para v1 comparado con el promedio general de las fincas que fue de 95,49, además mostraron menores valores en algunas de sus variables cuando se compararon con el promedio general de todas las fincas, estas variables fueron v3,v4,v5,y v10 esta situación permite definir a este grupo como fincas de niveles de producción bajos donde no se presentan sistemas agroforestales solo monocultivos donde su ingreso total es bajo en este mismo grupo también se presentó un promedio mayor en la variables v6 y v8 con relación al promedio general lo cual indica que anqué en este grupo la cantidad de miembros de unidad familiar es alta sus ingresos, se ven reducidos por las bajas de la producción.

Aun así, los productores de estas fincas se mostraron dispuestos a implementar como alternativa los sistemas agroforestales aunque algunos se dedican a la extracción de madera entre las especies de

Tabla 2. Variables que explican los componentes principales en el Río Mejicano. Tumaco.

\begin{tabular}{lccccc}
\hline Variables & Componente 1 & Componente 2 & Componente 3 & Componente 4 & Componente 5 \\
\hline V1 & 10.000 & 0.6395 & 0.3486 & -.0523 & -.2230 \\
V3 & 0.6395 & 10.000 & 0.6687 & -.0103 & -.3496 \\
V4 & 0.3486 & 0.6687 & 10.000 & 0.0000 & -.3938 \\
V5 & 523 & -.0103 & 0.0000 & 10000 & -.1741 \\
V6 & 2230 & -.3496 & -3938 & -.1741 & 10.000 \\
V8 & 0.1845 & 0.1366 & -1621 & -.0344 & -.0069 \\
V12 & -.0536 & -.0976 & 0.1154 & -.1296 & -.1630 \\
\hline
\end{tabular}


mayor importancia son el cedro (Cedrella odorata) y el laurel (Cordia oliodora). Paredes (2001), consideró que los productores del río Mejicano consideran la extracción de madera como última alternativa para los ingresos familiares.

Castillo (1987), identificó las actividades productivas del Naya costero del pacifico colombiano en las que describe a la población del Naya preferentemente como agricultores de subsistencia, pescadores artesanales, corteros de madera, mineros en pequeña escala $\mathrm{y}$, en menor medida, cazadores.

El segundo grupo: Conformado por 12 fincas, este grupo presentó un promedio menor en las variables v1 $(69,00)$, v3 $(0)$, v4 $(0)$, v5 $(0)$ y v8 $(4,66)$ con relación al promedio general, pero un promedio alto en las variables v6, $(151,416)$ y v10 $(333,33)$ con relación al promedio total de v6 que fue de $(139,98)$ y el de v10 $(2,48)$. Aunque la producción de cacao v1 en este grupo, es mucho más alta que en el grupo 1 es relativamente baja con relación a la producción total de las fincas también carecen de sistemas agroforestales y se dedican al monocultivo de cacao como tal.

Una de las características más importantes de este grupo es lo alto que son sus ingresos no obstante lo que les entra sale de igual manera, pues los costos de producción son altos.

Angulo (1992), en la caracterización de los sistemas productivos en el río Mejicano encontró que el cultivo que más se presentaba como monocultivo sin asociación agroforestal era el coco,

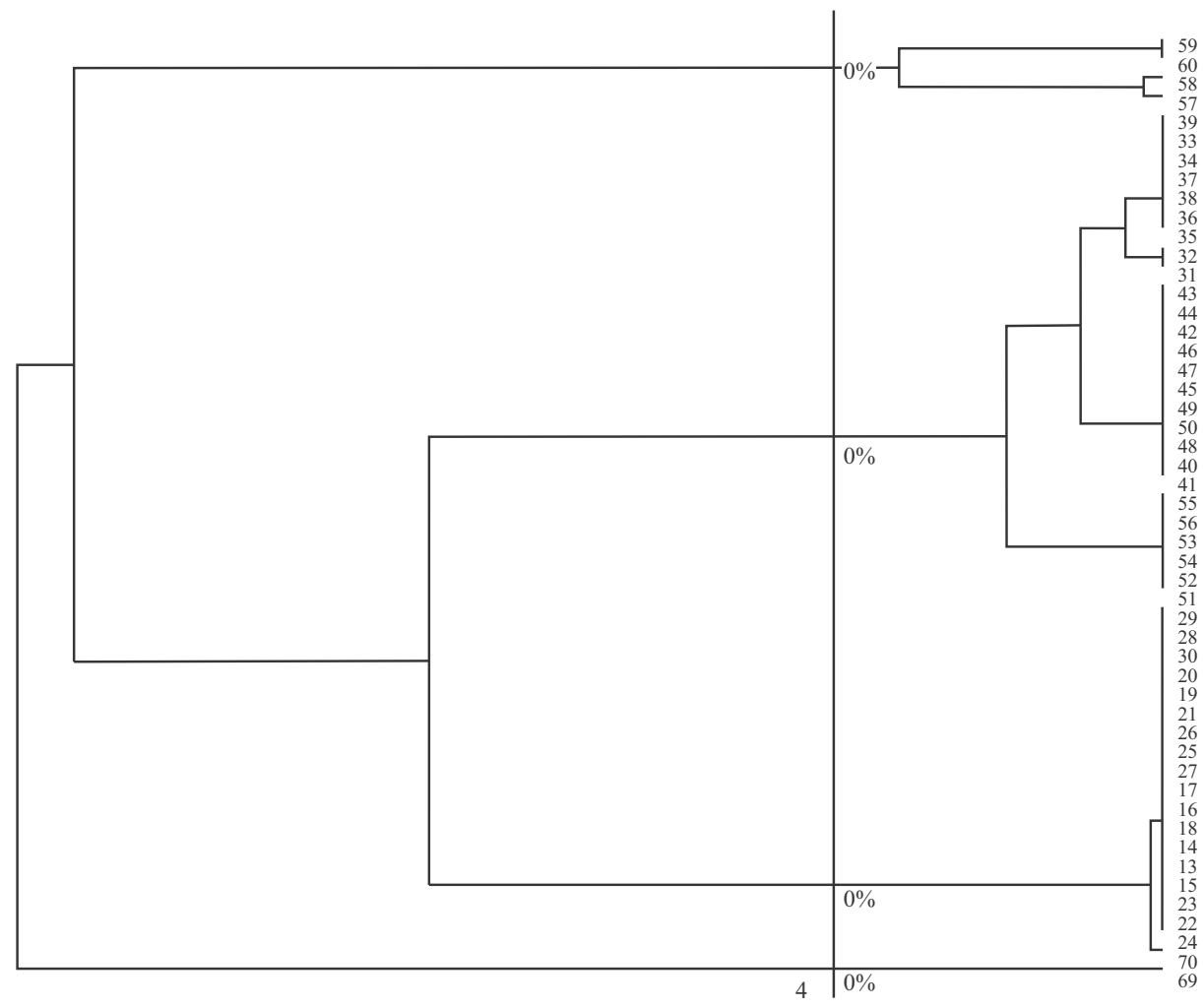

Figura 1. Dendrograma agrupación de fincas de los sistemas productivos del Rio Mejicano. 2018. 
destacándose por ser estable y por general ingresos altos.

El tercer grupo: estuvo formado por 20 fincas, este grupo se caracterizó por un promedio menor de las variables v1,(91.95), v6 (107.0) y v8 4,55 con relación al promedio total de v1 $(95,49)$ v6 $(139,98)$ y v8 $(5,64)$ y un promedio alto en las variables v3(1.45), v4(1.95),v5(9) y v10(250.000) con relación al promedio general que fue de para V3(0,92), V4(0,83), V5(2,25) y v10(248.90), las fincas agrupadas presentan sistemas agroforestales, sistemas silvopastoril y un ingreso total alto; pese a la baja producción de cacao este grupo, también es el único que presenta un componente pecuario a los productores de este grupo se podría trabajar con sistemas agroforestales compuestos por cacao y maderables

Paredes (2001), no identificó los sistemas silvopastoriles como uno de los cuatro sistemas que más se presentan en el río Mejicano

El cuarto grupo: estuvo formado por 15 fincas que se caracterizaron por las variables v1 $(156,66), \mathrm{v} 3$ $(2,26), \mathrm{v} 4(1,4), \mathrm{v} 8(7,2)$ fueron superiores al promedio total. Mientras que las variables v8 $(7,2) \mathrm{y}$ v10 (220.000) fueron inferiores al promedio total. De este grupo se resalta que presenta una producción de cacao alta posee hectáreas dedicadas a sistemas agroforestales y una población familiar baja, lo que lleva a contratar mano de obra y reduce sus ingresos.

En sistemas de producción la baja inversión de mano de obra en cada una de las actividades productiva es notoria, por esta razón las especies cultivadas son importantes permitiendo la reproducción de la mano de obra familiar(Angulo, 1992).

\section{Análisis de correspondencia múltiple (ACM)}

El análisis de los valores propios permitió seleccionar un factor que explica el 37,35\% de las variables cualitativas.

Del análisis de contribuciones de las variables a la conformación de los ejes, se pudo establecer que las variables que más contribuyeron a la conformación del factor uno fueron v3 (23.0) sistemas agroforestales que conocen, v5 labores fundamentales $(15,1), \mathrm{v} 11$ tenencia de tierra $(18,3), \mathrm{v} 12$ nivel de escolaridad $(12,0)$, v13 especie de la finca que más abundada $(14,1)$. Debido al poco manejo silvicultural los cultivos de cacao están expuestos a enfermedades como Mano de Bruja (Taphrina kruchii) trayendo como consecuencia bajo rendimiento. (Corpoica 1996), encontró que los productores del río mejicano tienen limitantes fitosanitarios los cuales en términos y conceptos del productor causan pérdidas económicas considerables.

Los servicios de salud y de educación no se prestan adecuadamente lo que es una constante en las comunidades de la costa pacífica situación similar encontró Paredes (2001).

\section{Análisis de clasificación (ACM)}

El análisis de clasificación basado en las características cualitativas para las fincas del río Mejicano permitió la conformación de cuatro grupos bien definidos agrupados en cada clase.

El primer grupo: Conformado por 10 fincas que representan el 16,67\% de todas las fincas, en esta 
clase el $100 \%$ de las fincas poseen fertilidad alta (V1), el 100\% también posee como especie más abundante el cedro (V13-3). El 83\% de las fincas tienen implementado sistemas silvopastoriles. (V3-1), y el 33\% la enfermedad que más abunda es mano de bruja (Taphrina kruchii)(v4).

El segundo grupo: está formado por 10 fincas que representan el 36,67\% las fincas que conforman este grupo se caracterizan por tener implementación de cercas vivas con un porcentaje de 100\% (V3-2), suelos de fertilidad media 44\% (V1-2). A diferencia del grupo 1en este grupo el Plátano (Musa sapientum) con un (75,78\%)(V13-1).

El grupo tres: Está formado por 10 fincas que representan $(41,67)$ del total de las fincas, el 100\% presenta árboles dispersos (V3-4), 100\% tenencia de tierra es en compañía (V11-2), 83,33\% (V4-2) enfermedad madura biche $85,71 \%$ (V13-2) especie que más abunda (Musa sapientum) y 50\% (V12) fertilidad media.

El grupo cuatro: está formado por 3 fincas que representan $5.00 \%$ de variabilidad total, 100\%(V113 ) en donde la tenencia de tierra arrendada, 100\% (V3-3) huertos mixtos, 100\% (V12-2) escolaridad regular y 75\% (V5-4) labores de manejo limpieza.

\section{Propuesta agroforestal para el río mejicano}

Actualmente los sistemas tradicionales agroforestales identificados en el río Mejicano presentan una complejidad de limitantes por su manejo técnico, el cual es muy tradicional e ineficiente, las cuales deben ser tenidas en cuenta en el momento de establecer un programa de fomento y capacitación.

Las principales limitantes de manejo técnico son: cultivos de cacao de mas de 20 años y con bajas densidades; árboles maderables en ocasiones muy dispersos y sin manejo silvicultural, falta de control fitosanitario, abundante presencia de maleza que favorece la proliferación de plagas y enfermedades en los diferentes cultivos, trayendo como consecuencia bajo rendimiento.

Lo anterior situación, está provocando que los campesinos abandonen los cultivos tradicionales para dedicarse a los cultivos de uso ilícito con las consecuencias sociales nefasta que esta actividad ilegal trae para las comunidades.

Para plantear un sistema agroforestal se tuvieron en cuenta las siguientes definiciones

El enfoque de sistemas: consiste en una estrategia de investigación fundamentada en el desarrollo de tecnología relevante y viable para los productores, la cual debe basarse en un conocimiento completo del sistema real de la finca, evaluando la tecnología no solamente en términos de desempeño técnico, sino también en términos de su identificación con las metas, necesidades y condiciones socioeconómicas del sistema de la finca así como la participación del productor como elemento central (Ruiz,1987.)

También se define como cualquier conjunto de elementos o componentes relacionados que interactúan entre sí (Leesberg 1987) o un arreglo de componentes físicos, o colección de cosas unidas o relacionadas de tal manera que forman y actúan como una unidad (Hart, 1985); un sistema es una estructura de componentes que interactúan entre sí con un entorno, para producir efectos previstos y 
avanzar en su adaptación (Hernández y Navia 1999).

De acuerdo a lo anterior se plantea un sistema de la siguiente manera:

Opción 1: Cacao (Theobroma cacao) - Plátano (Musa sapientum) - Cedro (Cedrela odorata)

Cacao (Theobroma cacao): El cacao trae benéficos al sistema en la parte ecológica atrae micro fauna y proporciona sombra, económica: tiene altas posibilidades de ser exportado o vendido en el mercado local y social es adaptable a los productores de la zona. Para cacao se utilizarían los siguientes clones: PATRÓN: IMC67, COPA: ICS 165, MON: 1 Estos clones son tolerantes Mano de bruja (Taphrina kruchii) enfermedad que se presenta en la zona. (Olegario 2008).

Plátano (Musa sapientum): Dentro de estos arreglos se considera el plátano como un cultivo transitorio y socio importante para el cacao, el cual cumple la función de proporcionar la sombra a los injertos de cacao por lo menos durante su etapa de instalación y crecimiento; igualmente debe cumplir un propósito económico para amortiguar los costos ocasionados por la siembra del cacao.

Cedro (Cedrela odorata): Fue la especie con mayor peso ecológico en la zona se puede realizar aprovechamientos pues su madera es de excelente calidad (figura 2)

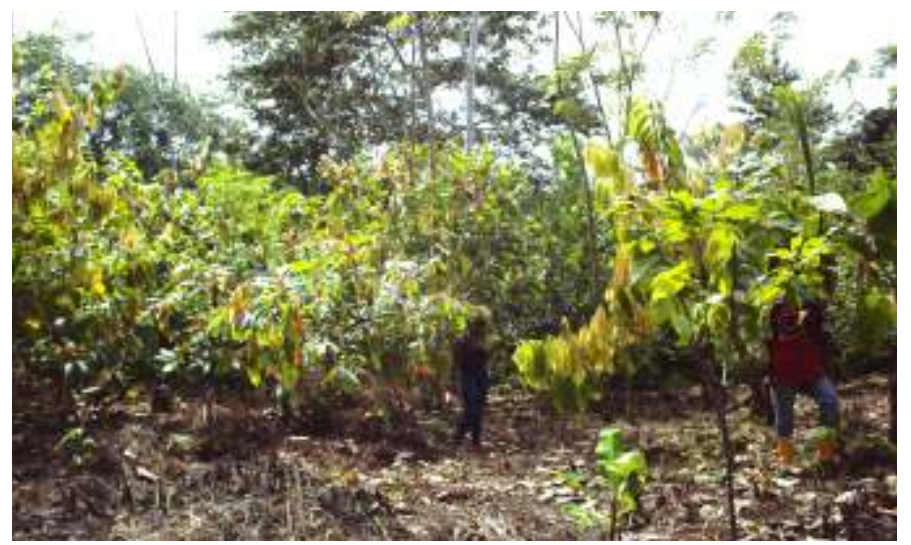

\section{Densidad de siembra} Plátano

Sembrado 3,5x3,5en triangulo 943 plantas

Cacao

Sembrado 3,5x3,5 en triangulo 943 plantas

Cedro

Sembrado 7x14: 102 plantas

Figura 2. Modelo agroforestal en San Andrés de Tumaco. Cacao-plátano, maderables (cedro). 2019.

\section{Labores de manejo}

Cacao (Theobroma cacao):

Al final del primer año de vida la planta de cacao se ramifica en forma de un verticilo compuesto de 2 a 5 ramas y llega el momento de hacerle la "poda de formación", que consiste en dejarle 3 - 4 ramas bien orientadas y distribuidas para que la planta se desarrolle erecta y en forma balanceada Todos los años la planta emite "chupones" que son ramas vegetativas, las cuales deberán ser eliminadas conjuntamente con las ramas sobrantes y secas.

Al mismo tiempo que se cortan los extremos de las ramas muy largas que tienden a caer al suelo .Se debe practicar también en la plantación la llamada "poda fitosanitaria", que tiene por finalidad eliminar todas las partes atacadas y/o dañadas por plagas, enfermedades o acción mecánica que se presentan en las plantas de cacao (ramas torcidas y/o desgarradas). Debe comprender también la recolección de frutos dañados o enfermos. Esto se puede hacer conjuntamente con las labores anteriores. 
Plátano (Musa sapientum).

Después de marcar los sitios en donde se va a sembrar y con suficiente anticipación, se procede a la apertura de los huecos, el tamaño es de 40 x 40 x $40 \mathrm{~cm}$. Es indispensable la preparación física y química del hueco, área donde se desarrollarán las raíces del plátano, ya que éstas son muy débiles y necesitan de suelo suelto para su desarrollo adecuado. Agregar 1 kilogramo de abono orgánico Mezclado con 100 gramos de cal magnesio y con la capa superficial del suelo, lo Cual facilita la permeabilidad, aireación y penetración de raíces.

Cedro (Cedrela odorata): Son importantes las limpias durante los primeros dos años. En caso de ataque, se recomienda la poda de la parte dañada, y cuando vienen los rebrotes, realizar una selección del mejor rebrote y eliminar los demás con tijeras podadoras. Esto evita la formación de bifurcaciones en la parte baja del árbol, que será la más valiosa desde el punto de vista. Maderable. (figura 3).

\begin{tabular}{|ccccccccccc}
$\mathrm{X}$ & 0 & $\mathrm{X}$ & 0 & $\mathrm{X}$ & 0 & $\mathrm{X}$ & 0 & $\mathrm{X}$ & 0 & $\mathrm{X}$ \\
& + & & & & + & & & & + & \\
& $\mathrm{X}$ & 0 & $\mathrm{X}$ & 0 & $\mathrm{X}$ & 0 & $\mathrm{X}$ & 0 & $\mathrm{X}$ & 0 \\
$\mathrm{X}$ & 0 & $\mathrm{X}$ & 0 & $\mathrm{X}$ & 0 & $\mathrm{X}$ & 0 & $\mathrm{X}$ & 0 & $\mathrm{X}$ \\
& $\mathrm{X}$ & 0 & $\mathrm{X}$ & 0 & $\mathrm{X}$ & 0 & $\mathrm{X}$ & 0 & $\mathrm{X}$ & 0 \\
$\mathrm{X}$ & 0 & $\mathrm{X}$ & 0 & $\mathrm{X}$ & 0 & $\mathrm{X}$ & 0 & $\mathrm{X}$ & 0 & $\mathrm{X}$ \\
& $\mathrm{X}$ & 0 & $\mathrm{X}$ & 0 & $\mathrm{X}$ & 0 & $\mathrm{X}$ & 0 & $\mathrm{X}$ & 0 \\
& + & & & & + & & & & + & \\
$\mathrm{X}$ & 0 & $\mathrm{X}$ & 0 & $\mathrm{X}$ & 0 & $\mathrm{X}$ & 0 & $\mathrm{X}$ & 0 & $\mathrm{X}$ \\
$7 * 14$ CEDRO & $3,5 * 3 * 5$ CACAO- PLATANO
\end{tabular}

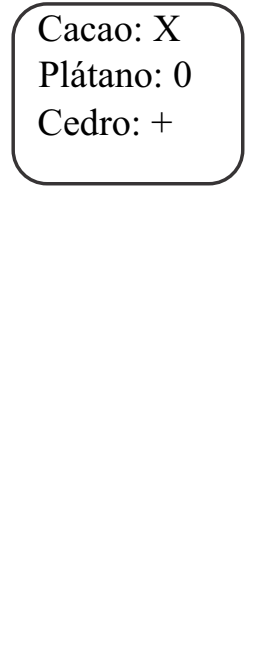

Figura 3. Arreglo agroforestal Cacao (Theobroma cacao), Plátano (Musa sapientum) y Cedro (Cedrela odorata).

\section{CONCLUSIONES}

El análisis de componentes principales (ACP) permitió identificar cuatro sistemas de producción formados con especies forestales (cedro), cultivos permanentes como (cacao y plátano) este sistema representa el $90 \%$ de productores del río y el 10\% presenta el mismo sistema con laurel como especie forestal.

El análisis de variables cualitativas (ACM), permitió agrupar las fincas con base a la fertilidad y manejo sanitario, presentando una limitante de tipo fitosanitario como la enfermedad mano de bruja (Taphrinakruchii). 
El sistema de producción del río Mejicano, está conformado por cultivos de subsistencia y auto consumo (cacao, plátano, coco, yuca y frutales), por cultivos forestales y algunas fincas tienen el componente pecuario (porcino, bovino y aves).

La región del río Mejicano, presenta una gran oferta natural de recursos de los cuales se destaca el cacao como uno de los componentes con mayor potencialidad de ser explotados.

\section{LITERATURA CITADA}

ANGULO, N. Evaluación de los sistemas productivos de los ríos: Mejicano, Gualajo, Tablones, Rosario y Chagüí, municipio de Tumaco, Nariño, 1992, 382p. Tesis de grado (Economista) Universidad de Nariño, Facultad de Ciencias Económicas.

CASTILLO, L. 1987. Actividades productivas del Naya costero del pacifico Colombiano. Cali: Universidad del Valle. 109 p.

CASTILlO M, L. E. 2002. Elementos de muetreo de poblaciones. México, Universidad Autónoma Chapingo, pp 37-62.

CORPORACION COLOMBIANA DE INVESTIGACION AGROPECUARIA. Caracterización de los sistemas producción en el Pacifico Colombiano Cali; CORPOICA, 1996.360p.

CORSETTI, M. Cambios tecnológicos, organizaciones sociales y actividades productivas predominantes en la Costa Pacifica Colombiana. Bogotá: Fondo FEN, 1990. 109 P.

GOBERNACIÓN DE NARIÑO. Cacao de Tumaco Nariño gana premio mundial a la excelencia en el Salón de Chocolate de París; 2015. Disponible en: http://nariño.gov.co/2012- 2015/index.php/prensa/6559-cacaode-tumaco-narino-gana-premio-mundial-a-laexcelencia-en-el-salon-de-chocolate-de-paris. Verificado julio 9 de 2019.

HART, R. Agro ecosistemas: conceptos básicos. Turrialba, Costa Rica: CATIE, 1984. 159 P.

HERNANDEZ, A. y NAVIA, J. Aspectos metodológicos del proceso de caracterización. En: informe técnico CORPOICA-SENA N ${ }^{\circ}$. Palmira. 1999.31P.

LEESBERG, J. y VALENCIA, E. Los sistemas de producción del otro medio chocoano. Bogotá: fondo FEM. 1992.121P.

NAVIA, J; y HERNANDEZ, A. Identificación de los sistemas de producción del Valle del Cauca documento de trabajo CORPOICA-SENA. Palmira 1999.

NAVIA, J; RESTREPO, J; VILLADA, D; OJEDA, P. Agroforestería: opción tecnológica para el manejo de suelos en zonas de ladera FIDAR, Santiago de Cali. 2003.

Parlamento Científico de jóvenes, 2018. Consultado 10 de Noviembre de 2017, en: https://parlamentocientificodejovenes.wordpress.com/clasificacion-de-los-sistemas-de-produccionagricola/ 
PAREDES, M. Identificación de los sistemas productivos del rio Mejicano del municipio de Tumaco, 2001, 160P. Trabajo de grado (ingeniero agrónomo) Universidad de Nariño. Facultad de Ciencias Agrícolas.

Plan de desarrollo municipal Tumaco 2008 - 2011. Diagnostico situacional de Tumaco Nariño. Consultado el 5 de marzo del 2018, en http://cdim.esap.edu.co/BancoMedios/Documentos\%20PDF/tumaco\%20\%20nari $\%$ C3\%B10\%20-\%20pd\%20-\%20diagnostico\%20-\%2008\%20-\%2011.pdf

Plan de Ordenamiento Territorial 2008 - 2019. Alcaldía municipal de Tumaco. Consultado el 1 de marzo del 2018, en http://cdim.esap.edu.co/BancoMedios/Documentos\%20PDF/tumaco-pot_2008_2019.pdf

PRECIADO, O. y OCAMPO, C. Caracterización del sistema tradicional de Cacao (Theobroma cacao) en la zona productiva del Municipio de Tumaco.

RUIZ, Á. Metodología del diagnostico de la producción de frijol. Cundinamarca. Bogotá: Universidad Nacional, 1997. 170p.

SANCHEZ, E. y LEAL, C. Elementos para una evaluación de sistemas productivos en el pacifico colombiano. Bogotá: biopacifico, 1994. 16P. 